\title{
Changes in Left Ventricular Size During Parabolic Flights by Two-dimensional Echocardiography and Level Set Method
}

\author{
C Corsi $^{1}$, G Saracino ${ }^{1}$, C Lamberti $^{1}$, S Cerutti $^{2}$, O Bailliart $^{3}$, B Cholley $^{3}$, A Capderou $^{4}, \mathrm{P} \mathrm{Vaida}^{5}$, \\ EG Caiani $^{2}$ \\ ${ }^{1}$ DEIS University of Bologna, Bologna, Italy \\ ${ }^{2}$ Politecnico di Milano, Dipartimento di Bioingegneria, Milano, Italy \\ ${ }^{3}$ CHU Lariboisière, Paris, France \\ ${ }^{4}$ Université Paris Sud, UPRES EA 2397, CCML, Physiologie, Le Plessis Robinson, France \\ ${ }^{5}$ Université Bordeaux 2, Médecine Aérospatiale, Bordeaux, France
}

\begin{abstract}
This study aims to evaluate changes on cardiac chambers size, induced by gravitational stresses. During parabolic flight, seven subjects underwent 2-D transthoracic echocardiography at three different gravity phases ( $1 \mathrm{Gz}, 1.8 \mathrm{Gz}$, and $0 \mathrm{Gz}$ ). LV endocardial borders were detected applying a semi-automatic segmentation procedure based on level set methods. LV cavity area was computed frame-by-frame for a whole cardiac cycle during each gravity phase. Expected modifications in $L V$ area with different gravity were found: at $1.8 \mathrm{Gz}$, enddiastolic (ED) and end-systolic (ES) areas were significantly $(p<0.05)$ reduced of $10.7 \pm 5.4 \%$ and $21.6 \pm 11.1 \%$ respectively, compared to $1 \mathrm{Gz}$ values, while they were increased of $11.2 \pm 5.4 \%$ and $11.1 \pm 6 \%$ during 0 Gz. Fractional area change was augmented of $20.9 \pm 29.1 \%$ at $1.8 \mathrm{Gz}$, while it remained unchanged at 0 $\mathrm{Gz}$, compared with $1 \mathrm{Gz}$ values. Furthermore, LV filling due to atrial contraction was increased at $0 \mathrm{Gz}$ of $39 \pm 35.6 \%$.
\end{abstract}

\section{Introduction}

Acute variations in gravity (head-to-foot acceleration, $\mathrm{Gz}$ ) induce dramatic fluid shifts in the body, as a consequence of changes in hydrostatic pressure gradients. Sudden exposure to microgravity causes an acute shift in fluid from the lower extremities toward the head and thorax, altering central filling volumes and pressures, resulting in significant cardiovascular effects [1,2]. These modifications, together with changes in the sympathetic activity (heart rate and inotropic status), influence left ventricular (LV) dimensions and function. These hemodynamic alterations [3] are responsible for many of the consequences associated with post-flight orthostatic intolerance in astronauts [1]. The comprehension of cardiovascular phenomena in a simulated microgravity environment is then crucial to investigate and understand physiological and pathologic changes occurring in space. Parabolic flights allow to carry out medical experiments on human subjects under conditions of weightlessness, complementing studies conducted on ground [4] and in space.

Echocardiography is a noninvasive, widespread and powerful diagnostic tool and because of its portability it can be utilized during parabolic flights conducted on specially configured aircraft. This imaging technique allows the visualization of the heart chambers and their modifications induced by gravitational stresses during parabolic flight.

The application of semi-automated image processing procedures to detect endocardial boundaries and extract LV dimensions can facilitate the data analysis and avoid the introduction of subjective interpretations. Among the border detection algorithms present in literature, level set methods are numerical techniques used to track the evolution of interfaces $[5,6]$, which have been applied to edge detection, shape recovery, representation and recognition [7-9].

Aim of this study was to test the feasibility to semiautomatically extract LV cavity dimensions from 2-D echo data obtained during different gravity conditions by means of level set techniques.

\section{Materials and methods}

\subsection{Subjects}

Seven healthy volunteers (mean age $\pm S D, 45 \pm 10$ ) were 
enrolled in this study. All subjects underwent special flight physical examination and were not taking any medication before and during flights. This study was approved from an ethical point of view by the French Space Agency (Centre National d'Etudes Spatiales (CNES)).

Subjects were studied in the upright position and to keep them in a near-standing position during microgravity, they were fastened to a saddle with a seat belt.

\subsection{Equipment and protocol}

Parabolic flights were performed in the Airbus A-300 Zero $G$ managed by Novespace for CNES and ESA in Bordeaux. Flights were managed on three consecutive days: each flight session lasted 2.5-3h and incorporated 30 parabolas.

Instantaneous gravity was measured continuously by using the aircraft's accelerometer. Gravity variations during a parabolic flight profile include four consecutive phases (Figure 1): normogravity (1 Gz), before the parabola began; mild hypergravity $(1.8 \mathrm{Gz})$ during the ascending phase of the parabola (20-25 sec); microgravity $(0 \mathrm{Gz})$, lasting for $20 \mathrm{sec}$ and corresponding to the top of the parabola; a second period of mild hypergravity $(1.8 \mathrm{Gz})$ during the descending phase of the parabola $(20-25 \mathrm{sec})$, followed by a new steady state at $1 \mathrm{Gz}(1 \mathrm{~min})$.

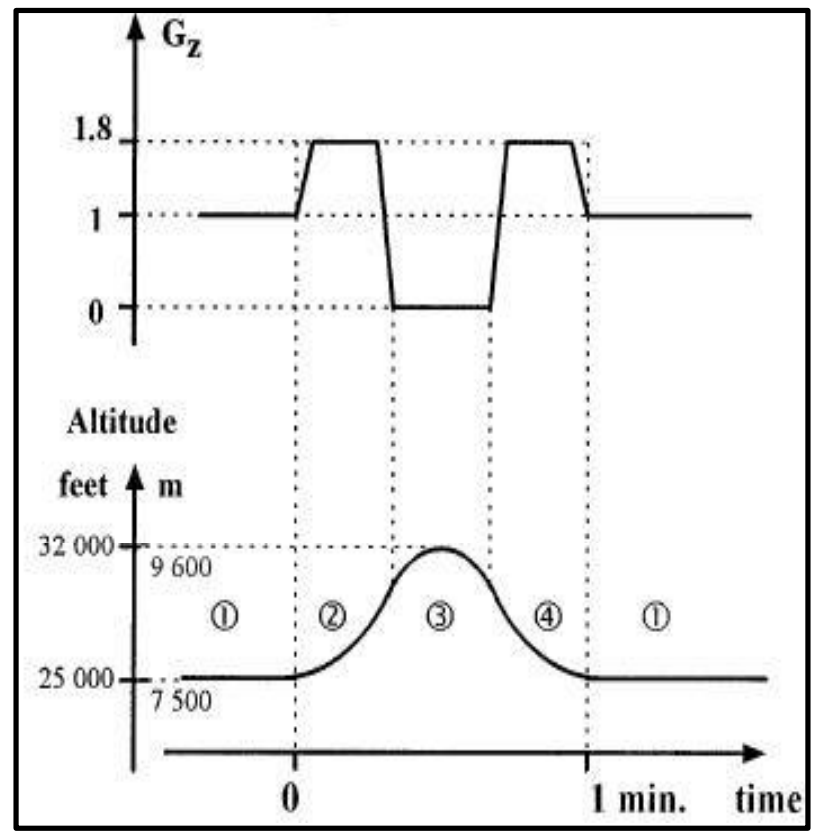

Figure 1. Schematic depicting different phases of parabolic flight profile and corresponding variations in gravity. Phase 1: normogravity $(1 \mathrm{Gz})$; phase 2 and 4: mild hypergravity $(1.8 \mathrm{Gz})$; phase 3 : microgravity $(0 \mathrm{Gz})$.

Ultrasound images were obtained (frame rate $37 \mathrm{~Hz}$ ) in the apical 4chamber view using an Aspen system (Acuson, France Siemens Cie, France), opportunely modified to digitally save on magneto-optical disk all the images relevant to the whole parabolic flight profile (about 1 minute of image acquisition per parabola).

\subsection{Level set methods}

Let's consider a $2 \mathrm{D}$ space and a curve $\gamma(\mathrm{t})$ that is propagating under the speed $F$ in the normal direction: $F$ indicates how to move each point of the curve. A level set function [5,6] is defined by taking the original curve $\gamma(\mathrm{t})$ and generating a surface that intersects the xy plane exactly where the curve $\gamma(t)$ sits. The intersection with the xy plane is called zero level set because it represents the set of points that are at height zero. To follow the deformations in $\gamma(t)$, the level set function is modified, so that the curve represents the moving zero level set of the level set function. The evolution of the level set function $\varphi(\mathrm{x}, \mathrm{t})$ is defined by a partial differential equation:

$$
\frac{\partial \varphi}{\partial t}+F|\nabla \varphi|=0
$$

with the initial condition $\varphi(\mathrm{x}, \mathrm{t})=\varphi_{0}$.

If $\gamma(\mathrm{t})=\varphi(0, \mathrm{t}), \gamma(\mathrm{t})$ moves with the desired speed function $F$, that is used to control shape recovery process in the image $I(\mathrm{x})[7]$ :

$$
F=g \varepsilon K-\beta \nabla g \cdot \frac{\nabla \varphi}{|\nabla \varphi|}
$$

with

$$
\begin{array}{r}
g(x)=\frac{1}{1+\left(\frac{\left|\nabla G_{\sigma} * I(x)\right|}{\alpha}\right)^{2}} \\
G_{\sigma}(\xi)=(1 / \sigma \sqrt{\pi}) e^{-(\xi / \sigma)^{2}}
\end{array}
$$

In (1), the first term on the right hand represents a surface tension force that depends on the Euclidean curvature $K$; the second term on the right hand describes a force that attracts the surface towards the boundaries, with a stabilizing effect. The edge indicator $g$ is a nonincreasing function of the gradient of a smoothed version of the initial image. $\alpha$ selects the contrast of the objects to be considered in the image during the motion of the embedding, while $\sigma$ is the variance of the gaussian determined by the size of the smallest features to be detected. The parameter $\beta$ is used to limit the regularization of the embedding controlled by the parameter $\varepsilon$.

The resulting equation of the motion for the level-set function $F(\mathrm{x}, \mathrm{t})$ is:

$$
\frac{\partial \varphi}{\partial t}+g \varepsilon K|\nabla \varphi|-\beta \nabla \varphi=0
$$




\subsection{Image and data analysis}

In each subject, for each gravity level (phase $1,1 \mathrm{Gz}$; phase $2,1.8 \mathrm{Gz}$; phase $3,0 \mathrm{Gz}$ ) images relevant to one complete cardiac cycle were selected and analyzed applying the modified level set model. For each frame, endocardial LV contour was detected and LV area measured as pixel counts. The reliability of the semiautomated contour identification was assessed by visual inspection during the analysis by an expert operator, by superimposing the detected contours on the original image, and allowing algorithm's parameters adjustment. LV function curves were obtained by plotting the area over time, and LV parameters were extracted: end-diastolic (EDA) and end-systolic (ESA) areas, respectively as the maximum and the minimum of the area in the cardiac cycle; fractional area change (FAC\%) as EDA-ESA normalized by EDA; atrial filling (AF), as the increase of LV area following the atrial contraction.

One-way analysis of variance for repeated measures was applied to test significant differences $(p<0.05)$ in $\mathrm{LV}$ parameters due to different gravity conditions.

\section{Results}

A total of 418 frames were analyzed: endocardial contours judged as reliable by an expert observer were automatically detected in $89 \%(371 / 418)$ of the processed frames, while the remaining $11 \%$ (47/418) needed further manual adjustment of the algorithm parameters. Local failures in the detection were associated with open mitral valves inclusion and apex or lateral wall misidentification, due to poor image quality. In figure 2 an example of the semi-automatically detected LV cavity contour is shown. The processing time was about 30 seconds per frame.

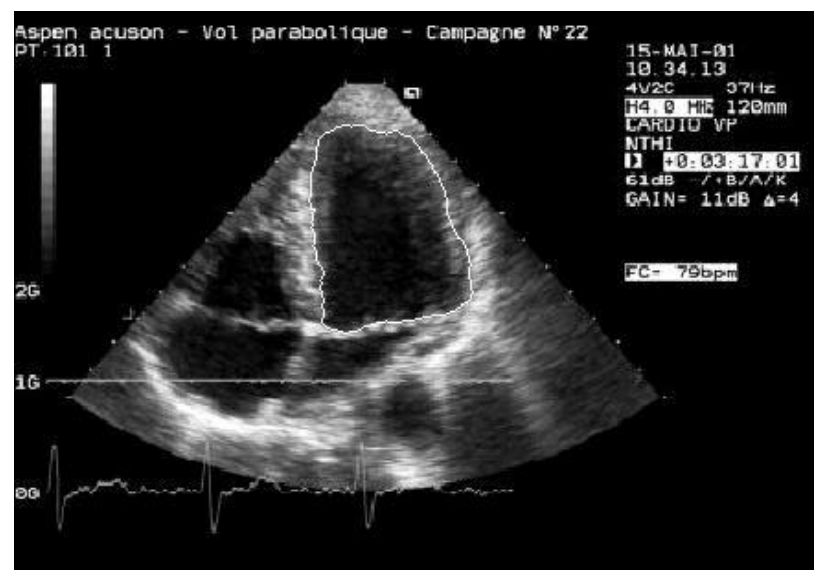

Figure 2. Example of an automatically detected LV endocardial contour superimposed to the original image at $1 \mathrm{Gz}$
In figure 3, the LV area versus time curves obtained during different $\mathrm{Gz}$ loads in one subject are shown. A reduction in $\mathrm{LV}$ area during $2 \mathrm{Gz}$ was visible, together with its increase during $0 \mathrm{Gz}$, compared to normogravity.

Table 1 shows the mean results in LV parameters obtained for the whole population, presented as percentages in respect to normogravity, i.e. to be assumed as $100 \%$. Expected modifications in LV area with different gravity were found: at $1.8 \mathrm{Gz}$, end-diastolic (EDA) and end-systolic (ESA) areas were significantly reduced of $10.7 \pm 5.4 \%$ and $21.6 \pm 11.1 \%$ respectively, compared to $1 \mathrm{Gz}$ values, while they were increased of $11.2 \pm 5.4 \%$ and $11.1 \pm 6 \%$ during $0 \mathrm{Gz}$. Fractional area change (FAC\%) was augmented of $20.9 \pm 29.1 \%$ at 1.8 $\mathrm{Gz}$, while it remained unchanged at $0 \mathrm{Gz}$, compared with $1 \mathrm{Gz}$ values. Furthermore, $\mathrm{LV}$ filling due to atrial contraction (AF) was increased at $0 \mathrm{Gz}$ of $39 \pm 35.6 \%$.

Table 1. Mean results, obtained in 7 subjects, of LV parameters (mean $\pm \mathrm{SD}$ ), expressed as percentage in respect to phase $1(1 \mathrm{Gz})(*$ : $\mathrm{p}<0.05$ in respect with $1 \mathrm{Gz})$.

\begin{tabular}{lcccc}
\hline & EDA & ESA & FAC $\%$ & AF \\
$1.8 \mathrm{Gz}$ & $91.7 \pm 8.5^{*}$ & $84.2 \pm 19.4^{*}$ & $120.9 \pm 29.1 *$ & $126 \pm 46.6$ \\
$0 \mathrm{Gz}$ & $111.2 \pm 5.4^{*}$ & $111.1 \pm 6.0 *$ & $100.7 \pm 8.9$ & $139 \pm 35.6^{*}$ \\
\hline
\end{tabular}

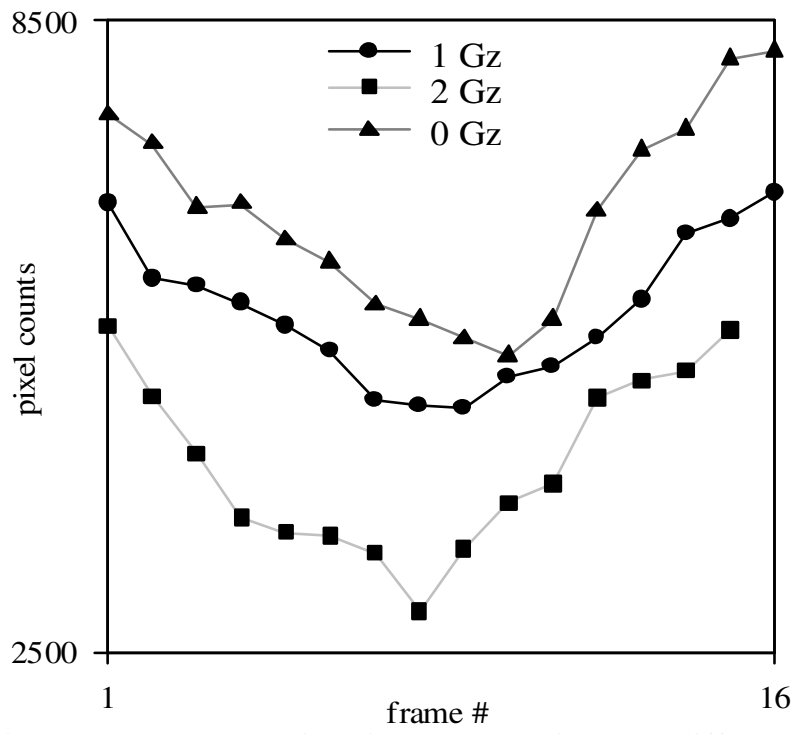

Figure 3. LV area (in pixels) over time at different gravity conditions (see legend in the figure).

\section{Discussion}

The results of this study confirmed that acute changes in venous return and cardiac loading conditions are 
induced by different gravity loads during parabolic flight.

The resulting variations in cardiac chambers size can be tracked using 2-D echocardiography. To analyze 2D echocardiographic images, a rapid, accurate and reproducible method for calculating $\mathrm{LV}$ dimensions is highly desirable. In clinical practice, LV area is usually calculated by manually tracing the endocardial contours but this technique is time-consuming and operatordependant.

Compared to this conventional technique, the applied algorithm, based on level set method, is faster and semi-automatic, requiring parameter's initialization on only one frame of the cardiac cycle. The endocardial detection was then automatic for the entire cineloop, by taking the estimated contour as initial condition for the contour detection of the following frame. In this way, the analysis can be extended to the whole cardiac cycle, instead of being limited to the telediastolic and telesystolic frames only, allowing the computation of the LV area over time and the extraction of LV function parameters. Expected modifications in LV area were found; interestingly, LV filling due to atrial contraction was doubled during microgravity, probably due to the increased venous return and subsequent atrial distension [10].

\section{Conclusions}

Despite the not excellent quality of the analyzed images, due to the critical experimental conditions, the proposed method was able to detect the dynamic changes in LV area caused by parabolic flights. Level set technique can be applied to 2-D ultrasound images for the semi-automatic extraction of LV endocardial contour and chamber dimensions.

\section{References}

[1] Lathers CM, Charles JB, Elton KF, Holt TA, Mukai C, Bennet BS, Bungo MW. Acute hemodynamic responses to weightlessness in humans. J Clin Pharmacol 1989;29:615-27.

[2] Norsk P, Foldager N, Bonde-Petersen F, Elmann-Larsen B, Johansen TS. Central venous pressure in humans during short periods of weightlessness. J Appl Physiol 1987;63:2433-7.

[3] Johns JP, Vernalis MN, Karemaker JM, Latham RD. Doppler evaluation of cardiac filling and ejection properties in humans during parabolic flight. J Appl Physiol 1994;76:2621-6.

[4] Carlson LD. Cardiovascular studies during and following simulation and weightlessness. Life Sci Space Res 1967;5:51-4.

[5] Osher S, Sethian JA. Fronts Propagating with Curvature Dependent Speed: Algorithms Based on Hamilton Jacobi formulation. Journal of Computational Physics 1988;79: 12-49.

[6] Sethian JA. Level set methods and fast marching methods. Cambridge:University Press 1999.

[7] Malladi R, Sethian JA, Vemuri BC. Shape Modelling with Front Propagation: A Level Set Approach. IEEE Trans. On Patterns Analysis and Machine Intelligence 1995;17(2):158-175.

[8] Sarti A, Lamberti C, Malladi R. Level set models for analysis of $2 \mathrm{D}$ and $3 \mathrm{D}$ echocardiographic data. In: Ravikanth Malladi, editor. Geometric Methods in BioMedical Image Processing. Springer Verlag, 2001:43-61.

[9] Corsi C, Saracino G, Sarti A, Lamberti C. Left Ventricular Volume Estimation for Real-Time ThreeDimensional Echocardiography. IEEE TMI Special Issue on New Trends on Cardiovascular Image Analysis 2002;21(9): in press.

[10] Videbaek R, Norsk P. Atrial distension in humans during microgravity induced by parabolic flights. J Appl Physiol 1997;83:1862-1866.

\author{
Address for correspondence. \\ Enrico G Caiani \\ Dipartimento di Bioingegneria, Politecnico di Milano \\ P.zza L. Da Vinci, 32, 20133 Milano, Italy. \\ E-mail: caiani@biomed.polimi.it
}

